\title{
Long-term survival results of patients with locally advanced gastric cancer and pathological complete response after neoadjuvant chemotherapy and resection
}

\author{
Tongbo Wang ${ }^{1}$, Nianchang Wang ${ }^{1}$, Hong Zhou ${ }^{1}$, Aiping Zhou ${ }^{2}$, Jing Jin ${ }^{3}$, Yingtai Chen ${ }^{1}$, Dongbing Zhao ${ }^{1}$ \\ ${ }^{1}$ Department of Pancreatic and Gastric Surgical Oncology, ${ }^{2}$ Department of Medical Oncology, ${ }^{3}$ Department of Radiation Oncology, National Cancer \\ Center/National Clinical Research Center for Cancer/Cancer Hospital, Chinese Academy of Medical Sciences and Peking Union Medical College, \\ Beijing 100021, China \\ Contributions: (I) Conception and design: T Wang, A Zhou, J Jin, Y Chen, D Zhao; (II) Administrative support: Y Chen, D Zhao; (III) Provision \\ of study materials or patients: T Wang, N Wang; (IV) Collection and assembly of data: T Wang, N Wang, H Zhou; (V) Data analysis and \\ interpretation: T Wang, N Wang; (VI) Manuscript writing: All authors; (VII) Final approval of manuscript: All authors. \\ Correspondence to: Yingtai Chen, MD, PhD; Dongbing Zhao, MD, PhD. Department of Pancreatic and Gastric Surgical Oncology, National Cancer \\ Center/National Clinical Research Center for Cancer/Cancer Hospital, Chinese Academy of Medical Sciences and Peking Union Medical College, \\ No. 17 Panjiayuan Nanli, Chaoyang District, Beijing 100021, China. Email: yingtaichen@126.com; dbzhao@cicams.ac.cn.
}

Background: To date, the long-term outcomes of patients with locally advanced gastric cancer (LAGC) who achieved a pathological complete response (pCR) after neoadjuvant therapy are elusive. To evaluate the impact of pCR on the long-term survival of LAGC patients who underwent neoadjuvant therapy and evaluate the necessity of postoperative adjuvant chemotherapy.

Methods: We conducted a retrospective study of clinicopathological and survival data of patients who achieved a pCR after neoadjuvant therapy and resection at the China National Cancer Center between January 2007 and December 2018.

Results: Ultimately, 39 patients enrolled in the current study, with a median follow-up time was 30.4 (range 2.5-101.6) months. The 3- and 5-year overall survival (OS) rates were $88.9 \%$ and $88.9 \%$, respectively. And the 3- and 5-year disease-free survival (DFS) rates were $88.9 \%$ and $88.9 \%$, respectively. During the followup, recurrence was observed in 3 patients. Of all 39 patients, $51.3 \%(\mathrm{n}=20)$ received postoperative adjuvant chemotherapy and $48.7 \%(\mathrm{n}=19)$ did not. There was no significant difference in OS $(\mathrm{P}=0.48)$ and DFS $(\mathrm{P}=0.47)$ between patients who underwent postoperative adjuvant chemotherapy and patients who did not.

Conclusions: Patients with LAGC who achieved a pCR after neoadjuvant therapy and resection might have a favorable OS and DFS. Our study failed to demonstrate the benefit of adjuvant chemotherapy for those patients.

Keywords: Gastric cancer; neoadjuvant therapy; pathological complete response (pCR); locally advanced gastric cancer (LAGC); postoperative adjuvant chemotherapy

Submitted Aug 11, 2019. Accepted for publication Nov 11, 2019.

doi: $10.21037 /$ tcr.2019.11.37

View this article at: http://dx.doi.org/10.21037/tcr.2019.11.37

\section{Introduction}

Gastric cancer is the third leading cause of cancerrelated deaths worldwide (1). At the time of diagnosis, two-thirds of patients with gastric cancer have locally advanced disease (2). Despite multimodality treatment using systemic chemotherapy, chemoradiation and surgery, locally advanced gastric cancer (LAGC) remains a clinical challenge: the 5 -year survival rates were less than $50 \%$ (3).

Neoadjuvant therapy, or the notion that the administration of therapeutic agents before a main treatment, has been 
recommended for treatment of patients with LAGC by most guidelines, including those of the National Comprehensive Cancer Network (NCCN) (4). However, the pathological complete response (pCR) rate of gastric or esophagogastric junction cancer after neoadjuvant chemotherapy is relatively low. To date, only a few sporadic case reports and a metaanalysis showed a certain survival benefit of patients with a pCR (5-16). Moreover, there is no evidence on whether the patients who achieved pCR should receive adjuvant chemotherapy or not.

As such, we performed a retrospective analysis to evaluate the impact of a pCR on the long-term survival of LAGC patients who underwent neoadjuvant therapy at China National Cancer Center. And we also evaluated the impact of adjuvant chemotherapy on survival of patients with pCR. To the best of our knowledge, this cohort is the largest to date in the literature for patients with LAGC achieved pCR after neoadjuvant therapy.

\section{Methods}

\section{Selection of patients}

We retrospectively reviewed the patients with LAGC treated with neoadjuvant therapy who achieved a pCR at the China National Cancer Center between January 2007 and December 2018. The inclusion criteria were as follows: (I) histologically confirmed of gastric adenocarcinoma by upper gastrointestinal endoscopy prior to treatment; (II) presence of locally advanced disease (cT3-4a and/or $\mathrm{N}+$ ) according to $7^{\text {th }}$ edition TNM system of American Joint Committee on Cancer; (III) administrated systemic chemotherapy or concurrent chemoradiation prior to surgery; (IV) histological grade of tumor regression after surgical resection was classified as complete regression based on Mandard tumor regression grade (TRG). The exclusion criteria included patients with non-adenocarcinoma histology, distant metastatic disease (M1) and incomplete clinical or pathological data registered. In addition, all patients included had an Eastern Cooperative Oncology Group performance status (PS) of 0-1. All study procedures were approved by the Institutional Review Board at the China National Cancer Center.

\section{Neoadjuvant therapy}

All patients received neoadjuvant therapy consisted of preoperative chemotherapy (nCT) or concurrent chemoradiation (nCCRT). All the patients were reviewed by a multidisciplinary team including gastrointestinal surgeons, radiologists, medical oncologists and radiation oncologists. Based on the patient's age, comorbidities, clinical TNM stage, and a pretreatment evaluation consisted of physical examination, complete blood count, hepatic function, serum tumor marker assessment and electrocardiogram, the most suitable therapeutic strategy was recommended. For patients who administrated preoperative chemotherapy, the regimens included FOLFOX (oxaliplatin/5-Fu/folinic acid), SOX (oxaliplatin/S-1), paclitaxel/oxaliplatin and DOS (docetaxel/oxaliplatin/S-1). For patients underwent preoperative concurrent chemoradiation, a total dose of 45 Gy was applied, using 25 fractions of 1.8 Gy within 5 weeks delivered concurrently with S-1 at $80 \mathrm{mg} / \mathrm{m}^{2}$.

The response evaluation was performed every two cycles of preoperative chemotherapy or at the end of concurrent chemoradiation. The tumor response was classified according to the Response Evaluation Criteria in Solid Tumors (RECIST version 1.1).

\section{Surgery and bistological examination}

All the patients underwent gastrectomy and standardized D2 lymph node dissection 4-6 weeks after the completion of neoadjuvant treatment. The extent of gastrectomy, totally or sub-totally depended on the site and size of the primary tumor.

The histological grade of tumor regression was classified based on the Mandard TRG (17). TRG 1 (complete regression) shows absence of residual cancer and fibrosis extending through the different layers of the esophageal wall; TRG 2 is characterized by the presence of rare residual cancer cells scattered through the fibrosis; TRG 3 features an increase in the number of residual cancer cells, but fibrosis still predominated; TRG 4 shows residual cancer outgrowing fibrosis; and TRG 5 is characterized by absence of regressive changes. A pCR was defined as TRG1 at the primary tumor site (T0) and no regional lymph node metastasis (N0).

\section{Postoperative adjuvant chemotherapy and follow-up}

Adjuvant chemotherapy was recommended for all the patients enrolled in the current study. However, 19 patients declined adjuvant chemotherapy due to various personal reasons.

After the completion of treatment, all the patients were 
Table 1 Baseline characteristics of patients

\begin{tabular}{|c|c|}
\hline Characteristic & No., \% \\
\hline Age, median [IQR] & 57 [49-64] \\
\hline Male & $28(71.8 \%)$ \\
\hline \multicolumn{2}{|l|}{ Tumor location } \\
\hline Upper stomach & $10(25.6 \%)$ \\
\hline Middle stomach & $11(28.2 \%)$ \\
\hline Lower stomach & $17(43.6 \%)$ \\
\hline Whole stomach & $1(2.6 \%)$ \\
\hline \multicolumn{2}{|l|}{ Biopsy pathology } \\
\hline Well differentiated & $1(2.6 \%)$ \\
\hline Moderately differentiated & $3(7.7 \%)$ \\
\hline Poorly differentiated & 35 (89.7\%) \\
\hline \multicolumn{2}{|l|}{ Clinical T stage* } \\
\hline $\mathrm{T} 2$ & $2(5.1 \%)$ \\
\hline T3 & $10(25.6 \%)$ \\
\hline $\mathrm{T} 4$ & 27 (69.2\%) \\
\hline \multicolumn{2}{|l|}{ Clinical N stage* } \\
\hline No & $1(2.6 \%)$ \\
\hline $\mathrm{N} 1$ & $10(25.6 \%)$ \\
\hline N2 & $17(43.6 \%)$ \\
\hline N3 & $11(28.2 \%)$ \\
\hline
\end{tabular}

*, 7th edition TNM system of American Joint Committee on Cancer. IQR, interquartile range.

followed-up every 3 months for the first 3 years, every 6 months between 3 and 5 years, and every 1 year afterwards until death. Follow-up included physical examination, complete blood count, biochemical analysis, serum tumor marker assessment, chest and abdominopelvic computed tomography imaging and annual gastrointestinal endoscopy. The patient's survival status was obtained through telephonebased interviews as well as from the records of outpatient, with the follow-up period ending in March 2019.

\section{Statistics}

Continuous variables were expressed as median value and range or interquartile range (IQR). The overall survival (OS) is defined as the time from surgery to any cause of death, and the disease-free survival (DFS) is defined as the time from surgery to recurrence or metastasis. The KaplanMeier method was used to analyze the survival data.

The statistical analysis was performed with SPSS v25 (IBM Corp., Chicago, IL, USA), Stata v14 (StataCorp, College Station, TX, USA) and Graphpad Prism 7 (GraphPad Software, San Diego, California, USA).

\section{Results}

\section{Patient characteristics}

From January 2007 to December 2018, 39 patients were identified. Table 1 summarized the baseline of patient characteristics. The median age was 57 years (range, $49-64)$, and there were 28 males (71.8\%). The primary tumor was located in cardia of the stomach of 10 patients, in the fundus/body of 11 patients, in the antrum/pylorus of 17 patients and overlapping of the whole stomach of 1 patient. The histological grade of preoperative endoscopic biopsy was poorly differentiated adenocarcinoma in 35 patients, moderately differentiated in 3 patients and well differentiated in 1 .

\section{Neoadjuvant therapy and surgery}

Table 2 summarized the treatments of all the patients. Of the 39 patients, 27 patients received $\mathrm{nCT}$ and 12 patients underwent nCCRT. For 27 patients in the nCT group, chemotherapeutic schemes included FOLFOX $(\mathrm{n}=12)$, $\operatorname{SOX}(n=7)$, paclitaxel/oxaliplatin $(n=1)$ and DOS $(n=7)$. The median number of cycles administered was 4 (range, 2-10).

All the 39 patients underwent radical gastrectomy plus D2 lymph node dissection, including proximal gastrectomy in 4 patients, distal gastrectomy in 23 and total gastrectomy in 12 . All of the cases were R0 resection, and the median lymph node detection was $27 \pm 10.1$.

\section{Postoperative adjuvant therapy}

For all the patients included, $20(51.3 \%)$ received adjuvant chemotherapy, 13 patients with the regimen of platinum/ fluoropyrimidine, 4 with taxane based regimens and 3 with S-1. Nineteen patients (48.7\%) didn't administrated adjuvant chemotherapy after surgical resection.

\section{Patients' survival}

After a median follow-up of 30.4 months (range, 5.1- 
Table 2 Pre-surgical/surgical/post-surgical treatments

\begin{tabular}{lc}
\hline Characteristic & No. $\%$ \\
\hline NAT pattern & $27(69.2 \%)$ \\
Chemotherapy & $12(30.8 \%)$ \\
FOLFOX & $7(17.9 \%)$ \\
SOX & $1(2.6 \%)$ \\
Paclitaxel/oxaliplatin & $7(17.9 \%)$ \\
DOS & $12(30.8 \%)$ \\
Concurrent chemoradiation & $4[2-10]$ \\
Neoadjuvant chemotherapy cycles, median & \\
[range] & \\
Gastrectomy type & $4(10.3 \%)$ \\
Proximal gastrectomy & $23(59.0 \%)$ \\
Distal gastrectomy & $12(30.8 \%)$ \\
Total gastrectomy & $27 \pm 10.1$ \\
Number of resected lymph nodes, mean \pm SD & \\
Adjuvant chemotherapy & $13(33.3 \%)$ \\
No & $4(10.3 \%)$ \\
Platinum + fluoropyrimidine & $3(7.7 \%)$ \\
Taxane based & \\
\hline S-1 & \\
\hline
\end{tabular}

NAT, neoadjuvant therapy; FOLFOX, oxaliplatin/5-Fu/folinic acid; SOX, oxaliplatin/S-1; DOS, docetaxel/oxaliplatin/S-1; SD, standard deviation.



101.6 months), the 1-, 3- and 5-year OS rates of all the 39 patients were $96.9 \%, 88.9 \%$ and $88.9 \%$, respectively. The 1-, 3- and 5-year DFS rates were 94.2\%, 88.9\% and 88.9\%, respectively. The Kaplan-Meier curves of OS and PFS of 39 patients were shown in Figure 1.

Further survival analysis was performed between patients who underwent adjuvant chemotherapy $(n=20,51.3 \%)$ and who did not $(n=19,48.7 \%)$. During the follow-up, recurrence was observed in 1 patient who received adjuvant chemotherapy and 2 patients who did not receive (Table 3). The recurrence site included abdominal lymph nodes $(n=2)$ and pleura metastasis $(n=1)$. Of these 3 patients, 1 died of upper gastrointestinal bleeding during adjuvant treatment and the other 2 patients died of metastatic disease. No significant difference was found in OS and DFS between the two groups (OS, $\mathrm{P}=0.48$; DFS, $\mathrm{P}=0.47$; Figure 2).

\section{Discussion}

Although evidence shows that patients with resectable gastric or gastroesophageal junction cancer who achieved pCR after neoadjuvant chemotherapy can gain a better outcome than patients without pCR (18), long-term survival for the patients with pCR remains unclear. The present study showed that the 1-, 3-, 5-year OS rates of LAGC patients who achieved a pCR after neoadjuvant therapy were $96.9 \%, 88.9 \%$ and $88.9 \%$, respectively. And the $1-, 3-$, 5 -year DFS rates of these 39 patients were $94.2 \%, 88.9 \%$

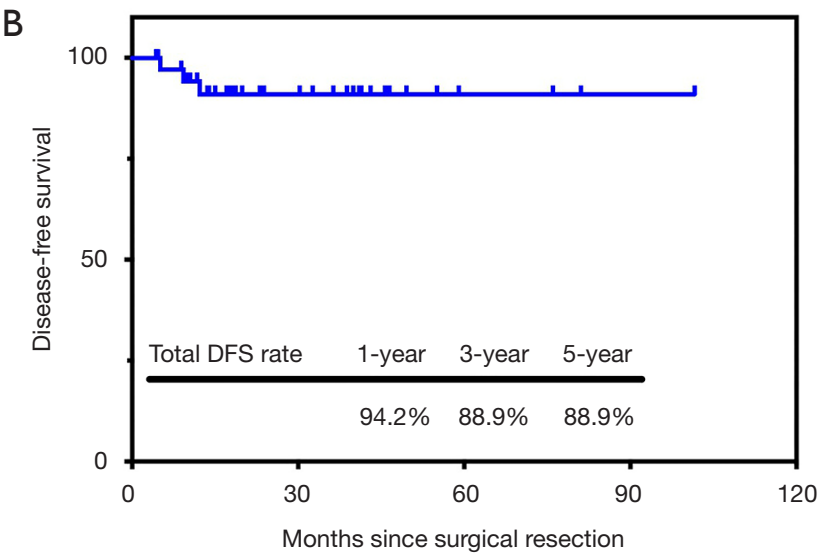

Figure 1 OS (A) and DFS (B) survival curves for all 39 patients with pCR after neoadjuvant therapy and resection. OS, overall survival; DFS, disease-free survival; pCR, pathological complete response. 
Table 3 Characteristics of patients who had developed recurrence

\begin{tabular}{llllcrc}
\hline Case No. & NAT & ACT & Recurrence Site & Cause of death & DFS (month) & OS (month) \\
\hline 1 & nCT & Yes & Abdominal LNs & Upper-Gl bleeding & 9.3 & 17.1 \\
2 & nCT & No & Pleura & Metastasis & 23.9 & 27.3 \\
3 & nCCRT & No & Abdominal LNs & Metastasis & 5.1 & 11.2 \\
\hline
\end{tabular}

NAT, neoadjuvant therapy; ACT, adjuvant chemotherapy; DFS, disease-free survival; OS, overall survival; nCT, neoadjuvant chemotherapy; nCCRT, neoadjuvant concurrent chemoradiation; LN, lymph node; Gl, gastrointestinal.
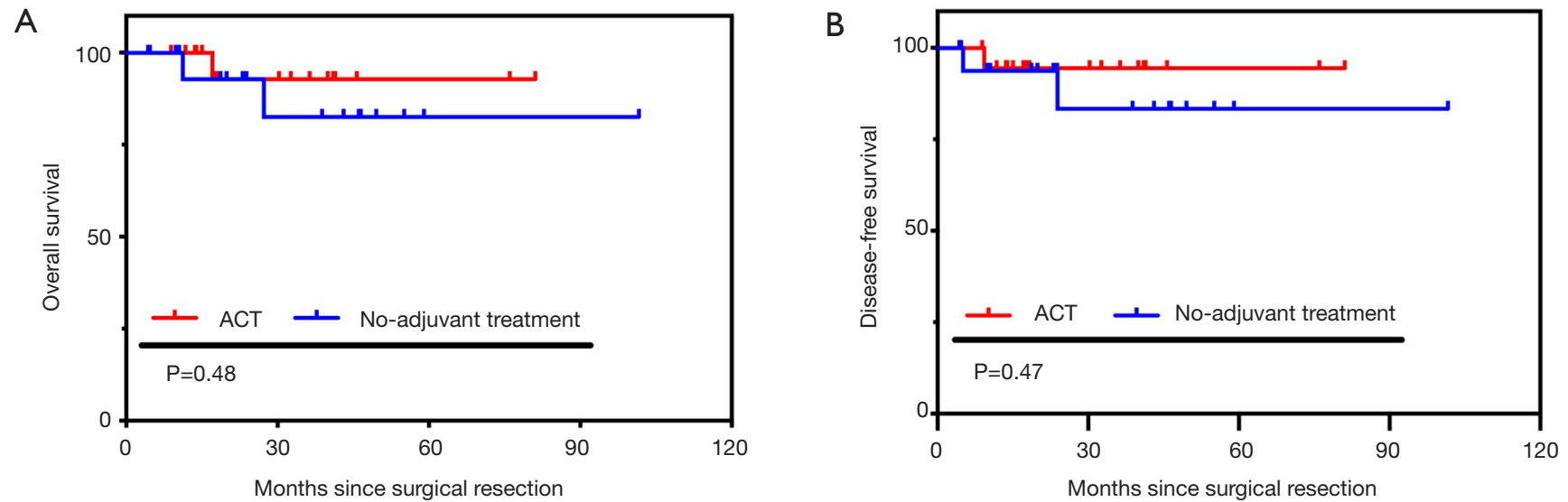

Figure 2 OS (A) and DFS (B) survival curves between patients with pCR who received adjuvant chemotherapy and who did not. OS, overall survival; DFS, disease-free survival; pCR, pathological complete response; ACT, adjuvant chemotherapy.

and $88.9 \%$, respectively. The survival rates seem slightly higher than a systematic review that reported a 5 -year OS of $85 \%$ and a recurrence-free survival of $75 \%$ among the patients with advanced gastric cancer who achieved a pCR after neoadjuvant therapy (19). A possible explanation is that the systematic review was based on published case reports which may have a publication bias.

To date, the evidence of the necessity of adjuvant chemotherapy for patients with pCR after neoadjuvant therapy is scarce. Moreover, in clinical practice, patients who achieved a pCR are less likely to undergo adjuvant chemotherapy than those with residual disease after neoadjuvant therapy and resection, just as shown in the present study $(51.3 \%$ with adjuvant chemotherapy $v s$. $48.7 \%$ without). Our subgroup analysis found that there was no significant difference in OS and DFS between patients who underwent adjuvant chemotherapy and patients who didn't, suggesting that adjuvant chemotherapy may not improve the survival of patients who achieved a pCR after neoadjuvant therapy. However, the modest sample size of the current study may not be capable of reaching a solid conclusion. Thus, future large population-based studies on this issue are needed.

Strengths and limitations should be considered when interpreting the study results. To the best of our knowledge, our study was the first original research that reported the long-term survival outcomes of the patients with LAGC who achieved a pCR after neoadjuvant therapy and resection. Secondly, the present study, for the first time, investigated the impact of adjuvant chemotherapy on the survival of patients with a pCR. While we had the largest number of the patients to date, the sample size was still modest therefore chance cannot be ruled out to draw a confirmative conclusion. Moreover, our follow-up was relatively short and few events were observed over this time. Finally, this was a single-institutional analysis with significant treatment heterogeneity.

\section{Conclusions}

Patients with LAGC who achieved a pCR after neoadjuvant therapy and resection might have a favorable OS and DFS. 
Our study failed to demonstrate the benefit of adjuvant chemotherapy for those patients.

\section{Acknowledgments}

Funding: This study received funding from the Special Research Projects for Capital Health Development, No. 2014-1-2151.

\section{Footnote}

Conflicts of Interest: The authors have completed the ICMJE uniform disclosure form (available at http://dx.doi. org/10.21037/tcr.2019.11.37). The authors have no conflicts of interest to declare.

Ethical Statement: The authors are accountable for all aspects of the work in ensuring that questions related to the accuracy or integrity of any part of the work are appropriately investigated and resolved. The study was conducted in accordance with the Declaration of Helsinki (as revised in 2013). This study was approved by the ethics committee of National Cancer Center/National Clinical Research Center for Cancer/Cancer Hospital, Chinese Academy of Medical Sciences and Peking Union Medical College (No. 17-156/1412). Informed consent was waived due to the retrospective nature of the study.

Open Access Statement: This is an Open Access article distributed in accordance with the Creative Commons Attribution-NonCommercial-NoDerivs 4.0 International License (CC BY-NC-ND 4.0), which permits the noncommercial replication and distribution of the article with the strict proviso that no changes or edits are made and the original work is properly cited (including links to both the formal publication through the relevant DOI and the license). See: https://creativecommons.org/licenses/by-nc-nd/4.0/.

\section{References}

1. Ferlay J, Soerjomataram I, Dikshit R, et al. Cancer incidence and mortality worldwide: sources, methods and major patterns in GLOBOCAN 2012. Int J Cancer 2015;136:E359-86.

2. Dong S, Yu JR, Zhang Q, et al. Neoadjuvant chemotherapy in controlling lymph node metastasis for locally advanced gastric cancer in a Chinese population. J Chemother 2016;28:59-64.
3. Nashimoto A, Akazawa K, Isobe Y, Gastric cancer treated in 2002 in Japan: 2009 annual report of the JGCA nationwide registry. Gastric Cancer 2013;16:1-27.

4. National Comprehensive Cancer Network. (NCCN) Clinical Practice Guidelines in Oncology. Gastric Cancer, Version 1, 2019. Available online: www.nccn.org

5. Suzuki Y, Kunisaki C, Tsuburaya A. A Case of Resected Advanced Gastric Cancer Exhibiting Pathological Complete Response after Neoadjuvant Chemotherapy(DTX/CDDP/S-1:DCS). Gan To Kagaku Ryoho 2015;42:2046-8.

6. Arisaka S, Kosaka R, Kunisaki C, et al. Pathological Complete Response of Advanced Gastric Cancer after Docetaxel and S-1 Combination Neoadjuvant Chemotherapy-A Case Report. Gan To Kagaku Ryoho 2016;43:889-92.

7. Kitayama K, Kanehara I, Teraoka H, et al. A case of advanced gastric cancer responding to S-1/CDDP neoadjuvant chemotherapy, leading to a pathological complete response. Gan To Kagaku Ryoho 2015;42:85-7.

8. Taniguchi $M$, Watanabe $Y$, Shirakawa $M$, et al. A case of advanced gastric cancer exhibiting pathological complete response after neoadjuvant chemotherapy. Gan To Kagaku Ryoho 2013;40:2298-300.

9. Watanabe T, Yoshikawa T, Kameda Y, et al. Pathological complete response of locally advanced gastric cancer after four courses of neoadjuvant chemotherapy with paclitaxel plus cisplatin: report of a case. Surg Today 2012;42:983-7.

10. Asaumi $Y$, Miyanaga T, Ito H, et al. Type 4 advanced gastric cancer responding to histological complete response after neoadjuvant S-1 combined with CDDP therapyreport of a case. Gan To Kagaku Ryoho 2011;38:1325-8.

11. Takasu N, Nomura T, Fukumoto T, et al. Advanced gastric cancer showing complete response to neoadjuvant chemotherapy with CPT-11 and S-1-a case report. Gan To Kagaku Ryoho 2009;36:111-3.

12. Mori S, Kishimoto H, Tauchi K, et al. Histological complete response in advanced gastric cancer after 2 weeks of S-1 administration as neoadjuvant chemotherapy. Gastric Cancer 2006;9:136-9.

13. Kobayashi N, Mizuta M, Otani H, et al. A case of locally advanced gastric cancer responding to pathological CR treated with S-1/CDDP neoadjuvant chemotherapy. Gan To Kagaku Ryoho 2010;37:1965-9.

14. Fujisawa T, Sano W, Ouchi S, et al. Complete histological response in gastric cancer stage IV after neoadjuvant chemotherapy including S-1 combined with CDDP-report of a case. Gan To Kagaku Ryoho 2007;34:2297-300. 
15. Oshima N, Tanizawa Y, Bando E, et al. Histological complete response in a case of advanced gastric cancer treated by neo-adjuvant chemotherapy with S-1/CDDP. Gan To Kagaku Ryoho 2010;37:697-701.

16. Wan T, Zhang XF, Liang C, et al. The Prognostic Value of a Pathologic Complete Response After Neoadjuvant Therapy for Digestive Cancer: Systematic Review and Meta-Analysis of 21 Studies. Ann Surg Oncol 2019;26:1412-20.

17. Mandard AM, Dalibard F, Mandard JC, et al. Pathologic assessment of tumor regression after preoperative chemoradiotherapy of esophageal carcinoma.

Cite this article as: Wang T, Wang N, Zhou H, Zhou A, Jin J, Chen Y, Zhao D. Long-term survival results of patients with locally advanced gastric cancer and pathological complete response after neoadjuvant chemotherapy and resection. Transl Cancer Res 2020;9(2):529-535. doi: 10.21037/tcr.2019.11.37
Clinicopathologic correlations. Cancer 1994;73:2680-6.

18. Li Z, Shan F, Wang Y, Zhang Y, et al. Correlation of pathological complete response with survival after neoadjuvant chemotherapy in gastric or gastroesophageal junction cancer treated with radical surgery: A metaanalysis. PLoS One 2018;13:e0189294.

19. Cho H, Nakamura J, Asaumi Y, et al. Long-term survival outcomes of advanced gastric cancer patients who achieved a pathological complete response with neoadjuvant chemotherapy: a systematic review of the literature. Ann Surg Oncol 2015;22:787-92. 